\title{
On the general sum-connectivity index and general Randić index of cacti
}

\author{
Shehnaz Akhter ${ }^{1}$, Muhammad Imran ${ }^{1,2^{*}}$ and Zahid Raza ${ }^{3}$
}

\begin{tabular}{l}
\hline${ }^{*}$ Correspondence: \\
imrandhab@gmail.com \\
'School of Natural Sciences (SNS), \\
National University of Sciences and \\
Technology (NUST), Sector H-12, \\
Islamabad, Pakistan \\
2Department of Mathematical \\
Sciences, United Arab Emirates \\
University, P.O. Box 15551, Al Ain, \\
United Arab Emirates \\
Full list of author information is \\
available at the end of the article
\end{tabular}

available at the end of the article

\begin{abstract}
Let $G$ be a connected graph. The degree of a vertex $x$ of $G$, denoted by $d_{G}(x)$, is the number of edges adjacent to $x$. The general sum-connectivity index is the sum of the weights $\left(d_{G}(x)+d_{G}(y)\right)^{\alpha}$ for all edges $x y$ of $G$, where $\alpha$ is a real number. The general Randić index is the sum of weights of $\left(d_{G}(x) d_{G}(y)\right)^{\alpha}$ for all edges $x y$ of $G$, where $\alpha$ is a real number. The graph $G$ is a cactus if each block of $G$ is either a cycle or an edge. In this paper, we find sharp lower bounds on the general sum-connectivity index and general Randić index of cacti.
\end{abstract}

MSC: 05C90

Keywords: cacti; general sum-connectivity index; general Randić index

\section{Introduction}

Let $G$ be a finite molecular graph of order $n$ and size $m$ with vertex set $V(G)$ and edge set $E(G)$. The degree of a vertex $x \in V(G)$, denoted by $d_{G}(x)$, is the number of edges adjacent to $x$. A vertex with degree 1 is called a pendent vertex. The minimum and maximum degrees of $G$ are respectively defined by $\triangle_{G}=\max \left\{d_{G}(x): x \in V(G)\right\}$ and $\delta_{G}=\min \left\{d_{G}(x)\right.$ : $x \in V(G)$ \}. The set of neighboring vertices of a vertex $x$ is denoted by $N_{G}(x)$. The graph obtained by deleting a vertex $x \in V(G)$ is denoted by $G-x$. The graph obtained from $G$ by adding an edge $x y$ between two nonadjacent vertices $x, y \in V(G)$ is denoted by $G+x y$.

Historically, the first degree based topological indices are the Zagreb indices, introduced by Gutman and Trinajestić [1]. The first Zagreb index $M_{1}(G)$ and the second Zagreb index $M_{2}(G)$ are defined as

$$
M_{1}(G)=\sum_{x \in V(G)}\left(d_{G}(x)\right)^{2} \quad \text { and } \quad M_{2}(G)=\sum_{x y \in E(G)} d_{G}(x) d_{G}(y) .
$$

The general Randić index (or product-connectivity index) was proposed by Bollobás and Erdős [2] and is defined as follows:

$$
R_{\alpha}(G)=\sum_{x y \in E(G)}\left(d_{G}(x) d_{G}(y)\right)^{\alpha}
$$

where $\alpha$ is a real number. Then $R_{-1 / 2}(G)=\sum_{x y \in E(G)}\left(d_{G}(x) d_{G}(y)\right)^{-\frac{1}{2}}$ is called the Randić index, which was defined by Randić [3], and $R_{1}$ is the second Zagreb index. The Randić index is the most studied, most applied, and most important index among all topologi-

(c) The Author(s) 2016. This article is distributed under the terms of the Creative Commons Attribution 4.0 International License (http://creativecommons.org/licenses/by/4.0/), which permits unrestricted use, distribution, and reproduction in any medium, provided you give appropriate credit to the original author(s) and the source, provide a link to the Creative Commons license, and indicate if changes were made. 
cal indices. Recently, Zhou and Trinajstić [4] modified the concept of Randić index and obtained a new index, called the general sum-connectivity index and defined as follows:

$$
\chi_{\alpha}(G)=\sum_{x y \in E(G)}\left(d_{G}(x)+d_{G}(y)\right)^{\alpha}
$$

where $\alpha$ is a real number. Then $\chi_{-1 / 2}(G)=\sum_{x y \in E(G)}\left(d_{G}(x)+d_{G}(y)\right)^{-\frac{1}{2}}$ is the sum-connectivity index defined by Zhou and Trinajstić [5], and if $\alpha=1$, then the general sumconnectivity index becomes the first Zagreb index.

A connected graph $G$ is a cactus if each block of $G$ is either a cycle or an edge. Let $X(n, t)$ be the set of cacti of order $n$ with $t$ cycles. Obviously, $X(n, 0)$ is the set of trees of order $n$, and $X(n, 1)$ is the set of unicyclic graphs of order $n$. Denote by $X^{0}(n, t)$ the $n$-vertex cactus consisting of $t$ triangles and $n-2 t-1$ pendent edges such that triangles and pendent edges have exactly one vertex in common.

Lin et al. [6] discuss the sharp lower bounds of the Randić index of cacti with $r$ pendent vertices. Dong and Wu [7] calculated the sharp bounds on the atom-bond connectivity index in the set of cacti with $t$ cycles and also in the set of cacti with $r$ pendent vertices, for all positive integral values of $t$ and $r$. Li [8] determined the unique cactus with maximum atom-bond connectivity index among cacti with $n$ vertices and $t$ cycles, where $0 \leq t \leq$ $\left\lfloor\frac{n-1}{2}\right\rfloor$, and also among $n$ vertices and $r$ pendent vertices, where $0 \leq r \leq n-1$. Ma and Deng [9] calculated the sharp lower bounds of the sum-connectivity index in the set of cacti of order $n$ with $t$ cycles and also in the set of cacti of order $2 n$ with perfect matching. Lu et al. [10] gave the sharp lower bound on the Randić index of cacti with $t$ number of cycles.

The present paper is motivated by the results of papers $[9,10]$. The goal of this paper is to compute the sharp lower bounds for the general sum-connectivity index and the general Randić index of cacti with fixed number of cycles.

\section{General sum-connectivity index}

In this section, we find sharp lower bound for the general sum-connectivity index of cacti. Let

$$
F(n, t)=2 t(n+1)^{\alpha}+(n-2 t-1) n^{\alpha}+4^{\alpha} t,
$$

where $n \geq 3$ and $t \geq 0$. First, we give some lemmas that will be used in the main result.

Lemma 2.1 ([4]) Let $n \geq 5$ be a positive integer, $\alpha<0$ be a real number, and $G \in X(n, 0)$. Then

$$
\chi_{\alpha}(G) \geq(n-1) n^{\alpha}
$$

Equality holds if and only if $G \cong X^{0}(n, 0)$.

Lemma 2.2 ([11]) Let $n \geq 5$ be a positive integer, $-1 \leq \alpha<0$ be a real number, and $G \in$ $X(n, 1)$. Then

$$
\chi_{\alpha}(G) \geq 2(n+1)^{\alpha}+(n-3) n^{\alpha}+4^{\alpha} .
$$

Equality holds if and only if $G \cong X^{0}(n, 1)$. 
Lemma 2.3 Let $x, k$ be positive integers with $x \geq k \geq 1$, and $\alpha<0$ be a real number. Define

$$
f(x)=x^{\alpha}-(x-k)^{\alpha}
$$

Then $f(x)$ is an increasing function.

Proof It is easily seen that $f^{\prime}(x)=\alpha\left(x^{\alpha-1}-(x-k)^{\alpha-1}\right)>0$ for $\alpha<0$. Therefore, $f(x)$ is an increasing function.

Lemma 2.4 Let $x, k$ be positive integers with $x \geq k \geq 1$, and $-1 \leq \alpha<0$ be a real number. Define

$$
f(x)=k(x+1)^{\alpha}+(x-k)(x+2)^{\alpha}-(x-k)(x+2-k)^{\alpha} .
$$

Then $f(x)$ is a decreasing function.

Proof Let $g(x)=k(x+1)^{\alpha}-k(x+2)^{\alpha}$ for $x \geq k \geq 1$. We get $g^{\prime}(x)=\alpha k\left((x+1)^{\alpha-1}-(x+2)^{\alpha-1}\right)<$ 0 . So, $g(x)$ is a decreasing function.

Let $h(x)=x(x+2)^{\alpha}$ for $x \geq k \geq 1$. Then $h^{\prime \prime}(x)=\alpha((\alpha+1) x+4)(x+2)^{\alpha-2}<0$ for $-1 \leq \alpha<0$. Hence, $h(x)-h(x-k)$ is a decreasing function. Note that $f(x)=g(x)+(h(x)-h(x-k))$. Thus, $f(x)$ is a decreasing function.

Lemma 2.5 Let $x, k$ be positive integers with $x \geq k \geq 1$, and $-1 \leq \alpha<0$ be a real number. Define

$$
f(x)=x(x+2)^{\alpha}-(x-2) x^{\alpha} .
$$

Then $f(x)$ is a decreasing function.

Proof Let $h(x)=x(x+2)^{\alpha}$ for $x \geq k \geq 1$. Then $h^{\prime \prime}(x)=\alpha((\alpha+1) x+4)(x+2)^{\alpha-2}<0$ for $-1 \leq \alpha<0$. But $f(x)=h(x)-h(x-2)$. Thus, $f(x)$ is a decreasing function.

Theorem 2.1 Let $-1 \leq \alpha<0$ be a real number, $n \geq 3$ be a positive integer, and $G \in X(n, t)$. Then

$$
\chi_{\alpha}(G) \geq F(n, t) .
$$

Equality holds if and only if $G \cong X^{0}(n, t)$.

Proof We use mathematical induction on $n$ and $t$. If $t=0$ or $t=1$, then Lemmas 2.1 and 2.2 give inequality (2.1). If $n=5$ and $t=2$, then there is only one possibility that $X^{0}(5,2)$ is a graph with two cycles that have only one common vertex (see Figure 1 ) and inequality (2.1) holds. If $n \geq 6$ and $t \geq 2$, then we will discuss the following two cases.

Case 1: $G \in X(n, t)$ has at least one pendent vertex.

Let $x \in V(G)$ be a pendent vertex and adjacent with vertex $y$ of degree $d$, where $2 \leq$ $d \leq n-1$. The set of neighbors of $y$ in $G$ is $N_{G}(y) \backslash\{x\}=\left\{u_{1}, u_{2}, u_{3}, \ldots, u_{d-1}\right\}$. Without lost of generality, we assume that $u_{1}, u_{2}, u_{3}, \ldots, u_{k-1}$ are pendent vertices and $u_{k}, u_{k+1}, \ldots, u_{d-1}$ 
Figure $1 X^{0}(5,2)$.

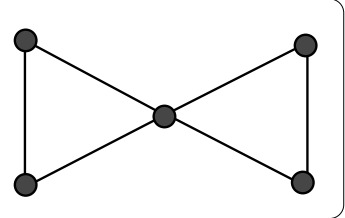

are nonpendent vertices, where $k \geq 1$. If $k=1$, then $u_{1}, u_{2}, u_{3}, \ldots, u_{d-1}$ are nonpendent vertices. Obviously, we have $t \leq\left\lfloor\frac{n-k-1}{2}\right\rfloor$. If $H$ is a graph obtained by deleting vertices $x, u_{1}, u_{2}, \ldots, u_{k-1}$ from $G$, that is, $H=G-x-u_{1}-u_{2}-\cdots-u_{k-1}$, then $H \in X(n-k, t)$. By inductive assumption and using Lemmas 2.3 and 2.4, we obtain

$$
\begin{aligned}
\chi_{\alpha}(G)= & \chi_{\alpha}(H)+k(d+1)^{\alpha}+\sum_{i=k}^{d-1}\left(\left(d+d_{G}\left(u_{i}\right)\right)^{\alpha}-\left(d-k+d_{G}\left(u_{i}\right)\right)^{\alpha}\right) \\
\geq & F(n-k, t)+k(d+1)^{\alpha}+\sum_{i=k}^{d-1}\left(\left(d+d_{G}\left(u_{i}\right)\right)^{\alpha}-\left(d-k+d_{G}\left(u_{i}\right)\right)^{\alpha}\right) \\
= & F(n, t)+2 t(n-k+1)^{\alpha}-2 t(n+1)^{\alpha}+(n-k-2 t-1)(n-k)^{\alpha}-(n-2 t-1) n^{\alpha} \\
& +k(d+1)^{\alpha}+\sum_{i=k}^{d-1}\left(\left(d+d_{G}\left(u_{i}\right)\right)^{\alpha}-\left(d-k+d_{G}\left(u_{i}\right)\right)^{\alpha}\right) \\
\geq & F(n, t)+2 t(n-k+1)^{\alpha}-2 t(n+1)^{\alpha}+(n-k-2 t-1)(n-k)^{\alpha}-(n-2 t-1) n^{\alpha} \\
& +k(d+1)^{\alpha}+(d-k)\left((d+2)^{\alpha}-(d-k+2)^{\alpha}\right) \\
\geq & F(n, t)+2 t(n-k+1)^{\alpha}-2 t(n+1)^{\alpha}+(n-k-2 t-1)(n-k)^{\alpha}-(n-2 t-1) n^{\alpha} \\
& +k n^{\alpha}+(n-1-k)(n+1)^{\alpha}-(n-1-k)(n+1-k)^{\alpha} \\
= & F(n, t)+(n-1-k-2 t)\left((n+1)^{\alpha}-(n-k+1)^{\alpha}-n^{\alpha}+(n-k)^{\alpha}\right) \\
\geq & F(n, t) .
\end{aligned}
$$

The equality holds if and only if $d=n-1,2 t=n-k-1$, and $H \cong X^{0}(n-k, t)$. Therefore, we have that $\chi_{\alpha}(G)=F(n, t)$ if and only if $G \cong X^{0}(n, t)$.

Case 2: $G \in X(n, t)$ has no pendent vertex. Then we consider edges $x_{0} x_{1}, x_{0} x_{2} \in E(G)$ such that $x_{0}$ and $x_{1}$ have degree 2 and $x_{2}$ has degree $d$, where $d \geq 3$. Now there arise two subcases.

Subcase 2.1: $x_{1}$ and $x_{2}$ are nonadjacent vertices in $G$. Then we construct a new graph $H$ by deleting a vertex $x_{0}$ and adding an edge between $x_{1}$ and $x_{2}$, that is, $H=G-x_{0}+x_{1} x_{2} \in$ $X(n-1, t)$. Then by inductive assumption we have

$$
\begin{aligned}
\chi_{\alpha}(G) & =\chi_{\alpha}(H)+(d+2)^{\alpha}+4^{\alpha}-(d+2)^{\alpha} \\
& \geq F(n-1, t)+4^{\alpha} \\
& =F(n, t)+2 t n^{\alpha}-2 t(n+1)^{\alpha}+(n-2 t-2)(n-1)^{\alpha}-(n-2 t-1) n^{\alpha}+4^{\alpha} \\
& =F(n, t)+2 t\left(n^{\alpha}-(n-1)^{\alpha}\right)+(n-2 t-2)\left((n-1)^{\alpha}-n^{\alpha}\right)+4^{\alpha}-n^{\alpha} \\
& >F(n, t) .
\end{aligned}
$$

The last inequality holds if $n \geq 7$. If $n=6$, then $t \leq 2$, and we easily show the inequality. 
Subcase 2.2: $x_{1}$ and $x_{2}$ are adjacent in $G$. Then we construct a new graph $H$ by deleting two vertices $x_{0}$ and $x_{1}$, that is, $H=G-x_{0}-x_{1} \in X(n-2, t-1)$. Let the set of neighbors of $x_{2}$ be $N_{G}\left(x_{2}\right) \backslash\left\{x_{0}, x_{1}\right\}=\left\{u_{1}, u_{2}, \ldots, u_{d-2}\right\}$, where all $u_{1}, u_{2}, \ldots, u_{d-2}$ are nonpendent vertices. By inductive assumption and using Lemmas 2.3 and 2.5 , we obtain

$$
\begin{aligned}
\chi_{\alpha}(G)= & \chi_{\alpha}(H)+4^{\alpha}+2(d+2)^{\alpha}+\sum_{i=1}^{d-2}\left(\left(d+d_{G}\left(u_{i}\right)\right)^{\alpha}-\left(d-2+d_{G}\left(u_{i}\right)\right)^{\alpha}\right) \\
\geq & F(n-2, t-1)+4^{\alpha}+2(d+2)^{\alpha}+\sum_{i=1}^{d-2}\left(\left(d+d_{G}\left(u_{i}\right)\right)^{\alpha}-\left(d-2+d_{G}\left(u_{i}\right)\right)^{\alpha}\right) \\
= & F(n, t)+2(t-1)(n-1)^{\alpha}-2 t(n+1)^{\alpha}+(n-2 t-1)(n-2)^{\alpha}-(n-2 t-1) n^{\alpha} \\
& +(t-1) 4^{\alpha}-t 4^{\alpha}+4^{\alpha}+2(d+2)^{\alpha}+\sum_{i=1}^{d-2}\left(\left(d+d_{G}\left(u_{i}\right)\right)^{\alpha}-\left(d-2+d_{G}\left(u_{i}\right)\right)^{\alpha}\right) \\
= & F(n, t)+2(t-1)(n-1)^{\alpha}-2 t(n+1)^{\alpha}+(n-2 t-1)(n-2)^{\alpha}-(n-2 t-1) n^{\alpha} \\
& +(t-1) 4^{\alpha}-t 4^{\alpha}+4^{\alpha}+2(d+2)^{\alpha}+(d-2)\left((d+2)^{\alpha}-d^{\alpha}\right) \\
= & F(n, t)+2(t-1)(n-1)^{\alpha}-2 t(n+1)^{\alpha}+(n-2 t-1)(n-2)^{\alpha}-(n-2 t-1) n^{\alpha} \\
& +d(d+2)^{\alpha}-(d-2) d^{\alpha} \\
= & F(n, t)+2(t-1)(n-1)^{\alpha}-2 t(n+1)^{\alpha}+(n-2 t-1)(n-2)^{\alpha}-(n-2 t-1) n^{\alpha} \\
& +(n-1)(n+1)^{\alpha}-(n-3)(n-1)^{\alpha} \\
= & F(n, t)+(n-1-2 t)\left((n+1)^{\alpha}-(n-1)^{\alpha}+(n-2)^{\alpha}-n^{\alpha}\right) \\
\geq & F(n, t) .
\end{aligned}
$$

The equality holds if and only if $d=n-1,2 t=n-1$, and $H \cong X^{0}(n-2, t-1)$. Therefore, we have $\chi_{\alpha}(G)=F(n, t)$ with equality if and only if $G \cong X^{0}(n, t)$.

\section{General Randić index}

In this section, we find sharp lower bound for the general Randić index of cacti. Let

$$
F_{1}(n, t)=2^{\alpha+1} t(n-1)+t 4^{\alpha}+(n-1-2 t)(n-1)^{\alpha},
$$

where $n \geq 3$ and $t \geq 0$. First, we give some lemmas that will be helpful in the proof of the main result.

Lemma 3.1 ([12]) Let $n \geq 5$ be a positive integer, $\alpha<0$ be a real number, and $G \in X(n, 0)$. Then

$$
R_{\alpha}(G) \geq(n-1)^{\alpha+1}
$$

Equality holds if and only if $G \cong X^{0}(n, 0)$.

Lemma 3.2 ([13]) Let $n \geq 5$ be a positive integer, $-1 \leq \alpha<0$ be a real number, and $G \in$ $X(n, 1)$. Then 


$$
R_{\alpha}(G) \geq(n-3)(n-1)^{\alpha}+2^{\alpha}(n-1)^{\alpha}+4^{\alpha} .
$$

Equality holds if and only if $G \cong X^{0}(n, 1)$.

Lemma 3.3 Let $x, d, k$ be positive integers with $d \geq k \geq 1, x \geq 2$, and $\alpha<0$ be a real number. Define

$$
f(x)=x^{\alpha}\left(d^{\alpha}-(d-k)^{\alpha}\right) .
$$

Then $f(x)$ is an increasing function.

Proof It is easily seen that $f^{\prime}(x)=\alpha x^{\alpha-1}\left(d^{\alpha}-(d-k)^{\alpha}\right)>0$ for $\alpha<0$. Therefore, $f(x)$ is an increasing function.

Lemma 3.4 Let $x, k$ be positive integers with $x \geq k \geq 1$, and $-1 \leq \alpha<0$ be a real number. Define

$$
f(x)=k x^{\alpha}+2^{\alpha}(x-k) x^{\alpha}-2^{\alpha}(x-k)^{\alpha+1} .
$$

Then $f(x)$ is a decreasing function.

Proof Let $g(x)=k x^{\alpha}\left(1-2^{\alpha}\right)$ for $x \geq k \geq 1$. We get $g^{\prime}(x)=\alpha k x^{\alpha-1}\left(1-2^{\alpha}\right)<0$. So, $g(x)$ is a decreasing function.

Let $h(x)=2^{\alpha} x^{\alpha+1}$ for $x \geq k \geq 1$. Then $h^{\prime \prime}(x)=2^{\alpha}(\alpha+1) x^{\alpha-1}<0$ for $-1 \leq \alpha<0$. Hence, $h(x)-h(x-k)$ is a decreasing function. Note that $f(x)=g(x)+(h(x)-h(x-k))$. Thus, $f(x)$ is a decreasing function.

Lemma 3.5 Let $x, k$ be positive integers with $x \geq k \geq 1$, and $-1 \leq \alpha<0$ be areal number. Let

$$
f(x)=x^{\alpha+1}-(x-2)^{\alpha+1} .
$$

Then $f(x)$ is a decreasing function.

Proof Let $h(x)=x^{\alpha+1}$ for $x \geq k \geq 1$. Then $h^{\prime \prime}(x)=\alpha(\alpha+1) x^{\alpha-1}<0$ for $-1 \leq \alpha<0$. But $f(x)=h(x)-h(x-2)$. Thus $f(x)$ is a decreasing function.

Theorem 3.1 Let $-1 \leq \alpha<0$ be a real number, $n \geq 3$ be a positive integer, and $G \in X(n, t)$. Then

$$
R_{\alpha}(G) \geq F_{1}(n, t)
$$

Equality holds if and only if $G \cong X^{0}(n, t)$.

Proof We use mathematical induction on $n$ and $t$. If $t=0$ or $t=1$, then inequality (3.1) holds by Lemma 3.1 and Lemma 3.2, respectively. If $n=5$ and $t=2$, then there is only one graph $X^{0}(5,2)$, and the result for $X^{0}(5,2)$ is trivial. Now if $n \geq 6$ and $t \geq 2$, then we will consider the following two cases. 
Case 1: $G \in X(n, t)$ has at least one pendent vertex.

Let $x$ be a vertex that is adjacent only with vertex $y$. Then $y$ has degree $d$ with $2 \leq d \leq$ $n-1$. The set of neighbors of $y$ in $G$ is $N_{G}(y) \backslash\{x\}=\left\{u_{1}, u_{2}, u_{3}, \ldots, u_{d-1}\right\}$. Without lost of generality, we assume that $d_{G}\left(u_{j}\right) \geq 2$ for $k \leq j \leq d-1$, where $k \geq 1$, and the remaining neighboring vertices of $y$ are pendent vertices. If $k=1$, then $G$ has no pendent vertex. Obviously, we have $t \leq\left\lfloor\frac{n-k-1}{2}\right\rfloor$. If $H$ is a new graph obtained by deleting vertices $x, u_{1}, u_{2}, \ldots, u_{k-1}$, that is, $H=G-x-u_{1}-u_{2}-\cdots-u_{k-1}$, then $H \in X(n-k, t)$. By inductive assumption and using Lemmas 3.3 and 3.4, we obtain

$$
\begin{aligned}
R_{\alpha}(G)= & R_{\alpha}(H)+k d^{\alpha}+\sum_{j=k}^{d-1}\left(\left(d d_{G}\left(u_{j}\right)\right)^{\alpha}-(d-k)^{\alpha} d_{G}\left(u_{j}\right)^{\alpha}\right) \\
\geq & F_{1}(n-k, t)+k d^{\alpha}+\sum_{j=k}^{d-1}\left(\left(d d_{G}\left(u_{j}\right)\right)^{\alpha}-(d-k)^{\alpha} d_{G}\left(u_{j}\right)^{\alpha}\right) \\
= & F_{1}(n, t)+2^{\alpha+1} t(n-k+1)^{\alpha}-2^{\alpha+1} t(n-1)^{\alpha}+(n-k-2 t-1)(n-k-1)^{\alpha} \\
& -(n-2 t-1)(n-1)^{\alpha}+k d^{\alpha}+\sum_{j=k}^{d-1}\left(\left(d d_{G}\left(u_{j}\right)\right)^{\alpha}-(d-k)^{\alpha} d_{G}\left(u_{j}\right)^{\alpha}\right) \\
\geq & F_{1}(n, t)+2^{\alpha+1} t(n-k+1)^{\alpha}-2^{\alpha+1} t(n-1)^{\alpha}+(n-k-2 t-1)(n-k-1)^{\alpha} \\
& -(n-2 t-1)(n-1)^{\alpha}+k d^{\alpha}+(d-k)\left((2 d)^{\alpha}-2^{\alpha}(d-k)^{\alpha}\right) \\
\geq & F_{1}(n, t)+2^{\alpha+1} t(n-k+1)^{\alpha}-2^{\alpha+1} t(n-1)^{\alpha}+(n-k-2 t-1)(n-k-1)^{\alpha} \\
& -(n-2 t-1)(n-1)^{\alpha}+k(n-1)^{\alpha}+2^{\alpha}(n-1-k)(n-1)^{\alpha}-2^{\alpha}(n+1-k)^{\alpha+1} \\
= & F_{1}(n, t)+(n-1-k-2 t)\left(1-2^{\alpha}\right)\left((n-k-1)^{\alpha}-(n-1)^{\alpha}\right) \\
\geq & F_{1}(n, t) .
\end{aligned}
$$

The equality holds if and only if $H \cong X^{0}(n-k, t), d=n-1$, and $2 t=n-k-1$. Therefore, we have that $R_{\alpha}(G)=F_{1}(n, t)$ if and only if $G \cong X^{0}(n, t)$.

Case 2: If $G$ has no pendent vertex, then we consider $x_{0} x_{1}, x_{0} x_{2}$ edges of a cycle such that $x_{0}$ and $x_{1}$ are vertices of degree two and $d_{G}\left(x_{2}\right)=d$, where $d \geq 3$. Next, we discuss this in the following two subcases.

Subcase 2.1: If $x_{1}$ and $x_{2}$ are nonadjacent vertices in $G$, then $H$ is a new graph obtained by deleting $x_{0}$ and adding an edge $x_{1} x_{2}$, that is, $H=G-x_{0}+x_{1} x_{2} \in X(n-1, t)$. Therefore, we get

$$
\begin{aligned}
R_{\alpha}(G)= & R_{\alpha}(H)+(2 d)^{\alpha}+4^{\alpha}-(2 d)^{\alpha} \\
\geq & F_{1}(n-1, t)+4^{\alpha} \\
= & F_{1}(n, t)+2^{\alpha+1} t(n-2)^{\alpha}-2^{\alpha+1} t(n-1)^{\alpha}+(n-2 t-2)(n-2)^{\alpha} \\
& -(n-2 t-1)(n-1)^{\alpha}+4^{\alpha} \\
= & F_{1}(n, t)+2^{\alpha+1} t\left((n-2)^{\alpha}-(n-1)^{\alpha}\right)+(n-2 t-2)\left((n-1)^{\alpha}-n^{\alpha}\right) \\
& +4^{\alpha}-(n-1)^{\alpha} \\
> & F_{1}(n, t) .
\end{aligned}
$$


The last inequality holds if $n \geq 7$. If $n=6$, then $t \leq 2$, and we easily show the inequality.

Subcase 2.2: If $x_{1}$ and $x_{2}$ are connected by an edge, then $H=G-x_{0}-x_{1} \in X(n-2, t-1)$. Let the set of neighbors of $x_{2}$ be $N_{G}\left(x_{2}\right) \backslash\left\{x_{0}, x_{1}\right\}=\left\{u_{1}, u_{2}, \ldots, u_{d-2}\right\}$, where $u_{j}$ for $1 \leq j \leq$ $d-2$ are nonpendent vertices. By inductive assumption and using Lemmas 3.3 and 3.5, we obtain

$$
\begin{aligned}
R_{\alpha}(G)= & R_{\alpha}(H)+4^{\alpha}+2^{\alpha+1} d^{\alpha}+\sum_{j=1}^{d-2}\left(\left(d d_{G}\left(u_{j}\right)\right)^{\alpha}-(d-2)^{\alpha} d_{G}\left(u_{j}\right)^{\alpha}\right) \\
\geq & F_{1}(n-2, t-1)+4^{\alpha}+2^{\alpha+1} d^{\alpha}+\sum_{j=1}^{d-2}\left(\left(d d_{G}\left(u_{j}\right)\right)^{\alpha}-(d-2)^{\alpha} d_{G}\left(u_{j}\right)^{\alpha}\right) \\
= & F_{1}(n, t)+2^{\alpha+1}(t-1)(n-3)^{\alpha}-2^{\alpha+1} t(n-1)^{\alpha}+(n-2 t-1)(n-3)^{\alpha} \\
& -(n-2 t-1)(n-1)^{\alpha}+(t-1) 4^{\alpha}-t 4^{\alpha}+4^{\alpha}+2^{\alpha+1} d^{\alpha} \\
& +\sum_{j=1}^{d-2}\left(\left(d d_{G}\left(u_{j}\right)\right)^{\alpha}-(d-2)^{\alpha}+d_{G}\left(u_{j}\right)^{\alpha}\right) \\
= & F_{1}(n, t) 2^{\alpha+1}(t-1)(n-3)^{\alpha}-2^{\alpha+1} t(n-1)^{\alpha}+(n-2 t-1)(n-3)^{\alpha} \\
& -(n-2 t-1)(n-1)^{\alpha}+(t-1) 4^{\alpha}-t 4^{\alpha}+4^{\alpha}+2^{\alpha+1} d^{\alpha} \\
& +2^{\alpha}(d-2)\left(d^{\alpha}-(d-2)^{\alpha}\right) \\
= & F_{1}(n, t)+2^{\alpha+1}(t-1)(n-3)^{\alpha}-2^{\alpha+1} t(n-1)^{\alpha}+(n-2 t-1)(n-3)^{\alpha} \\
& -(n-2 t-1)(n-1)^{\alpha}+2^{\alpha} d^{\alpha+1}-2^{\alpha}(d-2)^{\alpha+1} \\
= & F_{1}(n, t)+2^{\alpha+1}(t-1)(n-3)^{\alpha}-2^{\alpha+1} t(n-1)^{\alpha}+(n-2 t-1)(n-3)^{\alpha} \\
& -(n-2 t-1)(n-1)^{\alpha}+2^{\alpha}(n-1)^{\alpha}-2^{\alpha}(n-3)^{\alpha} \\
= & F_{1}(n, t)+(n-1-2 t)\left(1-2^{\alpha}\right)\left((n-3)^{\alpha}-(n-1)^{\alpha}\right) \\
\geq & F_{1}(n, t) .
\end{aligned}
$$

The equality holds if and only if $H \cong X^{0}(n-2, t-1), d=n-1$, and $2 t=n-1$. Therefore, we have that $R_{\alpha}(G)=F_{1}(n, t)$ if and only if $G \cong X^{0}(n, t)$.

\section{Conclusion}

In this paper, we determined the sharp lower bounds for the general sum-connectivity index and the general Randić index of cacti with fixed number of cycles for $-1 \leq \alpha<0$. The general sum-connectivity index and general Randić index of cacti for other values of $\alpha$ remains an open problem. Moreover, some topological indices and polynomials are still unknown for cacti with fixed number of cycles and fixed number of pendent vertices.

\section{Authors' contributions}

The idea to obtain the sharp lower bounds for the general sum-connectivity index and general Randić index of cacti was proposed by Shehnaz Akhter. After several discussions, Shehnaz Akhter and Muhammad Imran obtained some sharp lower bounds. Muhammad Imran and Zahid Raza checked these bounds and suggested to improve them. The first draft 
was prepared by Shehnaz Akhter, which was verified and improved by Muhammad Imran and Zahid Raza. The final version was prepared by Shenaz Akhter and Muhammad Imran. All authors read and approved the final manuscript.

\section{Author details}

'School of Natural Sciences (SNS), National University of Sciences and Technology (NUST), Sector H-12, Islamabad, Pakistan. ${ }^{2}$ Department of Mathematical Sciences, United Arab Emirates University, P.O. Box 15551, Al Ain, United Arab Emirates. ${ }^{3}$ Department of Mathematics, College of Sciences, University of Sharjah, Sharjah, United Arab Emirates.

Received: 24 September 2016 Accepted: 16 November 2016 Published online: 22 November 2016

\section{References}

1. Gutman, I, Trinajstic, N: Graph theory and molecular orbitals. Total $\pi$-electron energy of alternant hydrocarbons. Chem. Phys. Lett. 17, 535-538 (1972)

2. Bollobás, B, Erdős, P: Graphs of extremal weights. Ars Comb. 50, 225-233 (1998)

3. Randić, M: On characterization of molecular branching. J. Am. Chem. Soc. 97, 6609-6615 (1975)

4. Zhou, B, Trinajstić, N: On general sum-connectivity index. J. Math. Chem. 47, 210-218 (2010)

5. Zhou, B, Trinajstić, N: On a novel connectivity index. J. Math. Chem. 46, 1252-1270 (2009)

6. Lin, A, Luo, R, Zha, X: A sharp lower of the Randić index of cacti with $r$ pendants. Discrete Appl. Math. 156, 1725-1735 (2008)

7. Dong, $\mathrm{H}, \mathrm{Wu}, \mathrm{X}$ : On the atom-bond connectivity index of cacti. Filomat 28, 1711-1717 (2014)

8. Li, J: On the ABC index of cacti. Int. J. Graph Theory Appl. 1, 57-66 (2015)

9. Ma, F, Deng, H: On the sum-connectivity index of cacti. Math. Comput. Model. 54, 497-507 (2011)

10. Lu, M, Zhang, L, Tian, F: On the Randić index of cacti. MATCH Commun. Math. Comput. Chem. 56, $551-556$ (2006)

11. Du, Z, Zhou, B, Trinajstić, N: Minimum general sum-connectivity index of unicyclic graphs. J. Math. Chem. 48, 697-703 (2010)

12. Hu, Y, Li, X, Yuan, Y: Trees with minimum general Randić index. MATCH Commun. Math. Comput. Chem. 52, 119-128 (2004)

13. Wu, B, Zhang, L: Unicyclic graphs with minimum general Randić index. MATCH Commun. Math. Comput. Chem. 54 455-464 (2005)

\section{Submit your manuscript to a SpringerOpen ${ }^{\ominus}$ journal and benefit from:}

- Convenient online submission

- Rigorous peer review

- Immediate publication on acceptance

- Open access: articles freely available online

- High visibility within the field

- Retaining the copyright to your article 